\title{
Heart Rate Variability in Response to the Recall of Attachment Memories
}

Pietro Zingaretti ${ }^{1}$, Guido Giovanardi ${ }^{2,3}$, Gianluca Cruciani ${ }^{1}$, Vittorio Lingiardi ${ }^{3}$, Cristina

Ottaviani $^{4,5}$, Grazia Fernanda Spitoni ${ }^{3,5}$

${ }^{1}$ Department of Psychology, PhD program in Behavioral Neuroscience, Sapienza University of Rome, Rome, Italy

${ }^{2}$ Department of Psychology, University of Campania, Luigi Vanvitelli, Caserta, Italy

${ }^{3}$ Department of Dynamic and Clinical Psychology, Sapienza University of Rome, Rome, Italy

${ }^{4}$ Department of Psychology, Sapienza University of Rome, Rome, Italy

${ }^{5}$ Santa Lucia Foundation (IRCCS Fondazione Santa Lucia), Rome, Italy

*Corresponding author

Pietro Zingaretti, Via dei Marsi, 78, 00185, Rome, Italy. Telephone +39.06.49917877; E-mail: pietro.zingaretti@uniroma1.it. 


\begin{abstract}
Internal Working Models (IWM) of the attachment system are the devices by which individuals modulate emotional responses and the Disorganized attachment pattern is characterized by the breakdown of such emotion regulation. Vagally-mediated heart rate variability (vmHRV) is an index of regulated and flexible emotional responding. The current study aims at investigating vmHRV in non-clinical adults ( $\mathrm{n}=59 ; 29$ males) with an Organized or Disorganized attachment pattern before, during, and after the recall of attachment memories via the Adult Attachment Interview (AAI). Whereas the Organized group exhibited a typical decrease in vmHRV during the AAI and a return to a baseline-like pattern after the interview, the Disorganized group unexpectedly showed a sustained increase in vmHRV during the AAI which also persisted during the recovery phase. Results suggest that while Organized face the recollection of attachment memories by showing the adaptive stress response characterized by parasympathetic withdrawal and immediate recovery, individuals with Disorganized attachment need to keep regulating their emotions effortfully both during and after the recall of potential traumatic events.
\end{abstract}

Keywords: Emotion Regulation; Heart Rate Variability; Adult Attachment Interview; Organized Attachment; Disorganized Attachment. 


\section{Introduction}

According to attachment theory (Bowlby, 1982), early experiences with caregivers shape "Internal Working Models" (IWM) of attachment that guide emotion regulation, the process by which individuals modulate emotional responses to internal and external stimuli (Thompson, 1994). IWMs are mental representations of the relationships with and of the responsiveness of attachment figures that remain fairly see throughout life, from early childhood to adolescence and adulthood (Cassidy, 1994; Main, Kaplan \& Cassidy, 1985).

Individuals with secure attachment representations have IWMs characterized by a sense of felt security, as well as open and flexible emotion expression. On the other hand, individuals with insecure-anxious and insecure-dismissing IWMs entail expectations that the caregiver will not be available or will respond negatively to requests of help and are respectively dominated by the maximization of the expression and the suppression or masking of negative emotions (Kobak, Cole, Ferenz-Gillies, Fleming \& Gamble, 1993; Mikulincer \& Shaver, 2016). Lastly, unresolved IWMs include contradictory and dramatic expectations related to attachment figures, conveying dissociated emotional dispositions (Liotti, 2004; Lyons-Ruth \& Jacobvitz, 1999).

In recent years, the influence of IWMs on emotion regulation has been the object of investigation of several psychophysiological studies, which mostly focused on skin conductance (e.g. Dozier \& Kobak, 1992; Roisman, Tsai, \& Chiang, 2004) and heart rate variability (HRV), a measure of the variability of the time periods between adjacent heart beats which reflects the dynamic interplay between the parasympathetic and the sympathetic nervous systems. High tonic vagally-mediated HRV (vmHRV) is an index of robust parasympathetic control (i.e., via the vagus nerve) on the heart and has been associated with effective self-regulation as well as adaptive and flexible responses to meet various situational demands (e.g., Thayer, Hansen, Saus-Rose, \& Johnsen, 2009). On the other hand, phasic HRV suppression represents the withdrawal of cardiac vagal control and the activation of the defensive system and has been conceptualized as a biomarker of stress (Park \& Thayer, 2014). 
Existing literature highlights dissimilarities in vmHRV in individuals with distinctive attachment patterns, when exposed to stressful attachment-related tasks. For instance, Maunder et al. (Maunder, Lancee, Nolan, Hunter, \& Tannenbaum, 2006) found an inverse association between avoidant (but not anxious) attachment and vmHRV both at baseline and under stress. Ablow et al. (Ablow, Marks, Feldman, \& Huffman, 2013) assessed vmHRV during exposure to infants' cry in a sample of primiparous women with different styles of attachment. Women with secure attachment showed a stronger reduction in vmHRV compared to women with dismissing attachment, who failed to reveal significant changes in levels of vmHRV. Moreover, individuals characterized by low-avoidant attachment showed greater vmHRV following imagination of an attachment figure compared to imagination of a non-attachment figure, whereas this effect did not appear in individuals with highavoidant attachment (Bryant \& Hutanamon, 2018).

Despite the recent attention towards this topic, studies that measured vmHRV during the Adult Attachment Interview (AAI; George, Kaplan \& Main, 1996; Hesse, 2008), which is the best validated instrument to assess adult attachment representations, are sparse and report no significant differences in HRV on the basis of attachment style (e.g. Beijersbergen, Bakermans-Kranenburg, Van IJzendoorn, \& Juffer, 2008; Dias, Soares, Klein, Cunha, \& Roisman, 2011; Roisman, Tsai, \& Chiang, 2004). Tininenko, Measelle, Ablow and High (2012) used the AAI to study differences in respiratory sinus arrhythmia, finding lower values for participants who reported greater distress during the interview.

Notably, none of these studies has taken in account the disorganized/unresolved dimension of attachment. This is somehow surprising, as individuals with disorganized or unresolved attachment appear to be characterized by a momentary breakdown in emotion regulation strategies when confronted with traumatic experiences, or by a more general and pervasive lack of coherence in attachment narratives (e.g., Lyons-Ruth \& Jacobvitz, 1999; Main, Goldwyn, \& Hesse, 2002). However, previous research acknowledged a high degree of covariation between disorganized/unresolved and insecure-preoccupied states of mind, and the preoccupied states of mind have been demonstrated to correlate with an increased HR (Roisman, 2007). Consistently, patients 
with dissociative symptoms characterized by unresolved attachment showed a dominance of sympathetic over parasympathetic nervous system influences on the heart (assessed by the ratio of low-to-high frequency band power) after the AAI (Farina, Speranza, Imperatori, Quintiliani, \& Marca, 2015).

The aim of the current study is to examine vmHRV before, during, and after the AAI in a nonclinical sample of individuals with Organized/Resolved and Disorganized/Unresolved attachment. In line with the Polyvagal Theory (Porges, 2007), according to which high resting vmHRV as well as HRV suppression when facing a stressor are both indices of adaptive social and emotional regulation, we hypothesized that individuals with a Disorganized/Unresolved attachment would exhibit lower vmHRV at baseline, during, and after the AAI compared to individuals with an Organized/Resolved state of mind with respect to their attachment. On the other hand, we expect the latter to show a significant vmHRV decrease from baseline to AAI, to then return back to baseline levels during the recovery phase.

\section{Materials and Methods}

\subsection{Participants}

Computation of sample size was based on the only existing study combining the use of HRV assessment and the Adult Attachment Interview, which showed adequate power to detect differences of large size (Farina et al., 2015; $n=26$ ). Given that such study included a sample of individuals with dissociative disorders, which is an infrequent clinical condition, we have opted for a sample double in size. Fifty-nine individuals recruited from the general population participated in the study (30 females; mean age $29.56 \pm 8.78$ years; all Caucasians). Participants were recruited by word of mouth and the use of flyers distributed in the university area (e.g., bookshops, cafeterias, and public library). Exclusionary criteria included: diagnosis of psychiatric disorders (current and/or past), diagnosis of hypertension or heart disease, any other disease or use of drugs/medications that might affect cardiovascular function, menopause, use of oral contraceptives during the previous 6 months, 
pregnancy or childbirth within the last 12 months. Information about annual income and years of education is reported in Table 1.

[Insert Table 1 about here]

\subsection{Procedure}

The protocol was approved by the local Ethics Committee and all participants gave written informed consent prior to the study. Participants were informed of the following restrictions: no caffeine, alcohol, nicotine, or strenuous exercise for 2 hours prior to the appointment. After being instrumented for vmHRV monitoring, participants completed the experimental protocol, which included a 5-min 'vanilla' baseline period (Jennings, Kamarck, Stewart, Eddy, \& Johnson, 1992) during which participants were asked to perform "connect the dots"; the AAI, which had an average duration of $81.17 \pm 16.28 \mathrm{~min}$; and a 5 -min recovery phase in which participant were just invited to rest. After the recovery phase, participants completed the self-report questionnaires, and were fully debriefed.

\subsection{Physiological Assessment}

Heart rate (HR) was recorded as beat-to-beat intervals in milliseconds using the Bodyguard 2 (Firstbeat) HR monitor (e.g., Parak et al., 2015). VmHRV was assessed by computing the root mean square of successive beat-to-beat interval differences (rMSSD), which reflects vagal regulation of HR and is less susceptible to respiratory influences (Penttilä et al., 2001; Task Force, 1996). From now on, vmHRV will be used to refer to rMSSD. Outlier and artifact detection as well as HRV analyses were performed using Kubios HRV software (Tarvainen, Niskanen, Lipponen, Ranta-Aho, \& Karjalainen, 2014).

\subsection{Adult Attachment Interview}

The AAI (George, Kaplan, \& Main, 1996) is a semi-structured clinical interview designed to 
assess an individual's current state of mind with respect to past caregiver-child attachment-related experiences (Hesse, 2008). The individual's strategy during the AAI (e.g., derogating or minimizing of attachment vs. valuing and rendering a balanced, coherent narrative despite positivity or negativity of actual experience) is supposed to reflect the quality or security of one's current state of mind with respect to attachment. Typically, one out of four possible main classifications is assigned to the most prominent state of mind throughout the interview as a whole: secure/autonomous (F), insecuredismissing (Ds), insecure-preoccupied (E), or "Cannot Classify" (CC), of which secure/autonomous is considered the most beneficial. When present, discussions of experiences of loss, abuse, or other potential trauma are scored for disorientation in reasoning or discourse and, when sufficiently marked, may lead to a primary classification of a disorganized/unresolved (U) state of mind. The AAIs were transcribed verbatim and were coded by two certified AAI coders according to the AAI coding and classification system (Main et al., 2002). Interrater agreement on the five attachment categories was $87 \%(\mathrm{kappa}=.75)$.

\subsection{Statistical analyses}

Data processing was performed using SPSS v. 22 (IBM). Effect sizes were quantified by Cohen's $d$. Two-sample $t$-test and chi-square were used to test for pre-existing differences between the two groups (Disorganized/Unresolved vs. Organized/Resolved) in age and sex distribution. Differences in vmHRV at baseline, during the AAI, and during recovery between participants with Secure and Insecure attachment were also assessed. Then, to test for differences between participants with Disorganized attachment and those with Organized attachment in cardiac responses to the AAI, a 2 $\times 3$ General Linear Model (GLM) with group (Disorganized/Unresolved vs. Organized/Resolved) as a between-subject variable and time (baseline, AAI, recovery) as a within-subject variable, was conducted on vmHRV.

\section{Results}


The dataset had no missing data. AAIs were coded as follows: 15 were classified as Disorganized/Unresolved ( $\mathrm{U}$ or $\mathrm{CC} ; 25.4 \% ; 10$ by females) and 44 were classified as Organized/Resolved $(\mathrm{O} / \mathrm{R} ; 74.6 \% ; 20$ by females) with respect to attachment. Indices of disorganized/unresolved responses were related to experiences of loss, abuse, or both regarding primary caregivers (parents or grandparents). Of these interviews, 11 received an Unresolved (U) classification, mostly related to an unresolved loss, 4 received a Cannot Classify (CC) classification, and 1 was given both an Unresolved and a Cannot Classify classification. In the group with Organized/Resolved attachment, 25 interviews were classified as Secure/Autonomous (F), 15 as Dismissing (Ds), and 4 as Preoccupied (E).

No differences in vmHRV emerged between individuals with Secure $(\mathrm{N}=33)$ and Insecure $(\mathrm{N}$ $=26)$ attachment at baseline $(t=-0.76 ; p=.448)$, during the AAI $(t=-1.13 ; p=.262)$, or during the recovery phase $(t=-0.59 ; p=.556)$.

The GLM model showed significant main effect of Time, $F(2,56)=3.20, p=.05$, and Time $\mathrm{X}$ Group interaction, $F(2,56)=3.74, p=.03$, but no main effect of Group. As to the main effect of Time, post-hoc comparisons showed that vmHRV decreased from baseline to the AAI (19.7 $\pm 4.9 \mathrm{vs}$. $19.3 \pm 3.2 p=.291, d=.107)$ and significantly increased from AAI to recovery $(19.3 \pm 3.2$ vs. 20.8 $\pm 4.3 p=.001, d=.382)$.

[Insert Figure 1 about here]

As to the Time X Group interaction, Figure 1 depicts that in the Disorganized group, vmHRV significantly increased from baseline to the AAI (18.2 $\pm 4.7 \mathrm{~ms}$ vs. $19.6 \pm 2.8, p=.048, d=.363)$ and did not change from the AAI to recovery $(19.6 \pm 2.8$ vs. $19.5 \pm 3.8, p=.936, d=.018)$, whereas in the Organized group vmHRV significantly decreased from baseline to the AAI (20.3 \pm 4.8 vs. $19.2 \pm$ $3.4, p=.028, d=.254)$ and then returned to baseline levels from the AAI to recovery $(19.2 \pm 3.4 \mathrm{vs}$. 
$21.2 \pm 4.4, p=.000, d=.501)$. No differences between Disorganized/Unresolved vs. Organized/Resolved groups emerged in vmHRV at baseline, during the AAI or during the recovery phase (see Table 1).

\section{Discussion}

To the best of our knowledge, this is the first study to examine vmHRV during the AAI in nonclinical adults, characterized by a Disorganized/Unresolved or Organized/Resolved state of mind with respect to attachment. As expected, Disorganized/Unresolved significantly differed from individuals with Organized/Resolved attachment in their vagal response to the AAI, suggesting the use of different strategies in order to respond to the recollection of early attachment memories.

Contrary to our hypothesis, whereas the Organized/Resolved group exhibited a decrease in vmHRV during the AAI and a return to a baseline-like pattern after the interview, unexpectedly, Disorganized/Unresolved group showed a sustained increase in vmHRV during the AAI that persisted during the recovery phase. Indeed, previous research shows that during the AAI, individuals characterized by a secure attachment are characterized by a lower increase in physiological arousal (indexed by skin conductance levels), compared to groups with different attachment styles (e.g., Roisman et al., 2004). Beijersbergen et al. (2008) investigated physiological reactivity in adolescents during the AAI and found that those with a dismissing attachment representation are characterized by a reduced HR reactivity (but no differences in vagally-mediated HRV) during the AAI, compared to those with secure attachment, thereby concluding that dismissing adolescents either effectively use a defensive strategy during the AAI or cope with it in a superficial manner.

In the present study, it seems that individuals with Disorganized attachment were actively regulating their emotions during the AAI, using either reappraisal or suppression as indicated by the increase in vmHRV (e.g., Appelhans \& Luecken, 2006; Ingjaldsson, Laberg, \& Thayer, 2003). This was not the case for individuals with Organized attachment, who showed the "normal" stress response when asked to recall (for about 1 hour) their attachment related memories. Individuals with Organized 
attachment, however, were immediately able to recover after the end of the AAI, as indicated by the significant increase in levels of vmHRV (up to baseline levels) during the recovery phase following the interview. It is clear that individuals with Organized attachment engaged in "the" adaptive response, which is to react when the source of stress is present, and then to recover when it disappears (e.g., Al'Absi, 2011; Laborde, Mosley, \& Thayer, 2017). For sake of clarity, we remind that we found no differences in terms of vagal response to the AAI between individuals with secure and insecure attachment, suggesting a similar physiological reactivity between the two groups.

On the other hand, individuals with Disorganized attachment had to put effort into regulating their emotions during the AAI. The increase in vmHRV during the AAI in individuals characterized by Disorganized attachment likely reflects an active attempt to suppress negative memories and regulate emotions, which requires an effortful recruitment of resources and persists during the recovery phase after the interview. Interestingly, when the association between attachment and vmHRV was studied in more ecological contexts outside the laboratory (i.e., after recent marital separation), highly avoidant people who showed increases in vmHRV across a series of divorcerelated mental activation tasks also showed improvements in their self-concept over three months, whereas highly avoidant people who showed decreases in vmHRV showed no improvement or a worsening in their self-concept (Sbarra \& Borrelli, 2013). Unfortunately, due to limited resources, it was not possible to track changes in vmHRV in relation to the specific contents of the interview, which would have been particularly informative for the interpretation of increases in vmHRV in individuals with Disorganized attachment as an indicator of effortful emotion regulation, an orientation response or a dissociative state (e.g., Hesse \& Van IJzendoorn, 1998). The use of only one measure of vmHRV throughout the AAI may have diluted significant differences, as indicated by the similar vmHRV values during the Interview in the Organized and Disorganized groups shown in Figure 1.

An unexpected finding was the lack of significant differences between the two groups at baseline, where one would expect to find lower HRV in individuals with Disorganized/Unresolved compared 
to those with Organized/Resolved attachment. It must be noted however, that the lack of a significant $p$-value was accompanied by mean differences in the expected direction, with an effect size that was almost medium in magnitude $(d=.44)$. The low-vmHRV levels in the Disorganized group may either indicate that the baseline did not represent a true resting period, or that these individuals are in a constant state of (tonic) reactivity.

The main limitation of the present study is the small sample size and its differences from normative samples in literature: in our sample individuals with Disorganized/Unresolved attachment composed $25.4 \%$ of the total, a higher percentage compared to normative data, which reports $18 \%$ of the population as having a disorganized/unresolved state of mind (Bakermans-Kranenburg \& van IJzendoorn, 2009). Secondly, the disorganized subsample was much smaller in size, which reduces the statistical power of our analyses and may have biased some results (e.g., the main effect of Time following the Organized pattern). Thus, results should be interpreted with caution until these findings have been replicated in larger samples. Another limitation concerning the sample size is the small number of participants in the sub-groups of the Organized group. Particularly, there were only four individuals with a Preoccupied attachment in our Organized sample. This issue did not allow us to explore possible differences between the Organized sub-groups in terms of physiological reactivity. It has to be noted however, that our main result did not change after removing those four participants from the analysis. Future researches should take this aspect into account and compare individuals with a Preoccupied attachment with Secure and Dismissing ones. Lastly, the Disorganized group was heterogeneous since it includes interviews characterized by unresolved losses (Unresolved) but also interviews that could not be classified because of the presence of marked indications of several states of mind (Cannot-Classify). As a further limitation, likely due to the non-clinical sample, the 'Disorganized' term in the current study refers mainly to individuals with unresolved loss, not unresolved abuse or trauma, and this may have weakened the results.

Regardless of these limitations, present results suggest that on one hand those with Organized attachment face the recollection of early attachment memories by showing the adaptive stress 
response characterized by parasympathetic withdrawal and immediate recovery; on the other hand, individuals with Disorganized/Unresolved attachment need to effortfully regulate their negative emotions both during and after the recall of potential traumatic events. 


\section{Author Note}

The authors would like to thank the subjects whose participation made this study possible, Prof. Julian Thayer for his valuable inputs to the data interpretation and discussion of the present manuscript, and Ludovica Zanini for her precious cooperation in the revision of this manuscript.

The authors state no declaration of interest and this research did not receive any specific grant from funding agencies in the public, commercial, or not-for-profit sectors.

The authors assert that all procedures contributing to this work comply with the ethical standards of the relevant national and institutional committees on human experimentation and with the Helsinki Declaration of 1975 , as revised in 2008. 


\section{References}

Ablow, J. C., Marks, A. K., Shirley Feldman, S., \& Huffman, L. C. (2013). Associations between first-time expectant women's representations of attachment and their physiological reactivity to infant cry. Child Development, 84(4), 1373-1391. DOI: 10.1111/cdev.12135.

Al'Absi, M. (2011). Stress and addiction: Biological and psychological mechanisms. London, UK: Academic Press, Elsevier.

Appelhans, B. M., \& Luecken, L. J. (2006). Heart rate variability as an index of regulated emotional responding. Review of General Psychology, 10(3), 229-240. doi:10.1037/1089-2680.10.3.229

Bakermans-Kranenburg, M. J., \& van IJzendoorn, M. H. (2009). The first 10,000 Adult Attachment Interviews: Distributions of adult attachment representations in clinical and non-clinical groups. Attachment \& Human Development, 11(3), 223-263.

https://doi.org/10.1080/14616730902814762

Beijersbergen, M. D., Bakermans-Kranenburg, M. J., Van IJzendoorn, M. H., \& Juffer, F. (2008). Stress regulation in adolescents: Physiological reactivity during the adult attachment interview and conflict interaction. Child Development, 79(6), 1707-1720.

Bowlby, J. (1982). Attachment and loss, vol. 1: Attachment. New York, NY: Basic Books Classics. (Original work published 1969)

Bryant, R. A., \& Hutanamon, T. (2018). Activating attachments enhances heart rate variability. PloS One, 13(2), e0151747. https://doi.org/10.1371/journal.pone.0151747.

Cassidy, J. (1994). Emotion regulation: Influences of attachment relationships. Monographs of the society for research in child development, 59(2-3), 228-249.

Dias, P., Soares, I., Klein, J., Cunha, J. P., \& Roisman, G. I. (2011). Autonomic correlates of attachment insecurity in a sample of women with eating disorders. Attachment \& Human Development, 13(2), 155-167. DOI: 10.1080/14616734.2011.554005.

Dozier, M., \& Kobak, R. R. (1992). Psychophysiology in attachment interviews: Converging evidence for deactivating strategies. Child Development, 63(6), 1473-1480. 
Farina, B., Speranza, A. M., Imperatori, C., Quintiliani, M. I., \& Marca, G. D. (2015). Change in heart rate variability after the Adult Attachment Interview in dissociative patients. J. Trauma \& Dissociation, 16(2), 170-180. http://doi.org/10.1080/15299732.2014.975309.

George, C., Kaplan, N., \& Main, M. (1996). Adult attachment interview. Unpublished manuscript, (3rd ed.). Department of Psychology, University of California, Berkley.

Hesse, E., \& van IJzendoorn, M. H. (1998). Parental loss of close family members and propensities towards absorption in offspring. Developmental Science, 1, 299-305.

Hesse, E. (2008). The Adult Attachment Interview: Protocol, method of analysis, and empirical studies. In Cassidy, J., \& Shaver, P. R. (Eds.), Handbook of attachment: Vol. 2. Theory, research, and clinical applications (2nd ed.) (pp. 552-598). New York: Guilford Press.

Ingjaldsson, J. T., Laberg, J. C., \& Thayer, J. F. (2003). Reduced heart rate variability in chronic alcohol abuse: relationship with negative mood, chronic thought suppression, and compulsive drinking. Biological Psychiatry, 54(12), 1427-1436. https://doi.org/10.1016/S00063223(02)01926-1.

Jennings, J. R., Kamarck, T., Stewart, C., Eddy, M., \& Johnson, P. (1992). Alternate cardiovascular baseline assessment techniques: vanilla or resting baseline. Psychophysiology, 29, 742-750. https://doi.org/10.1111/j.1469-8986.1992.tb02052.x.

Kobak, R. R., Cole, H. E., Ferenz-Gillies, R., Fleming, W. S., \& Gamble, W. (1993). Attachment and emotion regulation during mother-teen problem solving: A control theory analysis. Child Development, 64, 231-245. https://www.jstor.org/stable/1131448.

Laborde, S., Mosley, E., \& Thayer, J. F. (2017). Heart Rate Variability and Cardiac Vagal Tone in Psychophysiological Research - Recommendations for Experiment Planning, Data Analysis, and Data Reporting. Frontiers in psychology, 8, 213. https://10.3389/fpsyg.2017.00213.

Liotti, G. (2004). Trauma, dissociation and disorganized attachment: Three strands of a single braid. Psychotherapy: Theory, Research, Practice, Training, 41, 472-486. 
Lyons-Ruth, K., \& Jacobvitz, D. (1999). Attachment disorganization: Unresolved loss, relational violence, and lapses in behavioral and attentional strategies. In Cassidy, J., \& Shaver, P. R. (Eds.), Handbook of attachment: Theory, research, and clinical applications (pp. 520-554). New York, NY: Guilford Press.

Main, M., Goldwyn, R., \& Hesse, E. (2002). Adult attachment scoring and classification systems. [Version 7.1]. Unpublished manuscript, University of California.

Main, M., Kaplan, N., \& Cassidy, J. (1985). Security in infancy, childhood, and adulthood: A move to the level of representation. Monographs of the Society for Research in Child Development, 50, 66-104. https://www.jstor.org/stable/3333827.

Maunder, R. G., Lancee, W. J., Nolan, R. P., Hunter, J. J., \& Tannenbaum, D. W. (2006). The relationship of attachment insecurity to subjective autonomic function during standardized acute stress in healthy adults. Journal of Psychosomatic Research, 60, 283-290. doi:10.1016/j.jpsychores.2005.08.013.

Mikulincer, M., \& Shaver, P. R. (2016). Attachment in adulthood: Structure, dynamics, and change (2nd ed.). New York, NY: Guilford Press.

Parak, J., Tarniceriu, A., Renevey, P., Bertschi, M., Delgado-Gonzalo, R., \& Korhonen, I. (2015). Evaluation of the beat-to-beat detection accuracy of PulseOn wearable optical heart rate monitor. Proceedings of the 37th Annual International Conference of the IEEE Engineering in Medicine and Biology Society (EMBC) (pp. 8099-8102). Milan, IT: IEEE EMBC. https://doi.org/10.1109/EMBC.2015. 7320273.

Park, G., \& Thayer, J. F. (2014). From the heart to the mind: cardiac vagal tone modulates topdown and bottom-up visual perception and attention to emotional stimuli. Frontiers in Psychology, 5, 278. https://doi.org/10.3389/fpsyg.2014.00278.

Penttilä, J., Helminen, A., Jartti, T., Kuusela, T., Huikuri, H. V., Tulppo, M. P., . . S Scheinin, H. (2001). Time domain, geometrical and frequency domain analysis of cardiac vagal outflow: 
Effects of various respiratory patterns. Clinical Physiology, 21 (3), 365-376.

https://doi.org/10.1046/j.1365-2281.2001.00337.x.

Porges S.W. (2007). The polyvagal perspective. Biological Psychology 74:116-143.

Roisman, G. I. (2007). The psychophysiology of adult attachment relationships: Autonomic reactivity in marital and premarital interactions. Developmental Psychology, 43: 39-53. DOI: 10.1037/0012-1649.43.1.39.

Roisman, G. I., Tsai, J. L., \& Chiang, K. S. (2004). The emotional integration of childhood experiences: Physiological, facial expressive, and self-reported emotional response during the Adult Attachment Interview. Developmental Psychology, 40, 776-789. DOI: 10.1037/00121649.40.5.776.

Sbarra, D. A., \& Borelli, J. L. (2013). Heart rate variability moderates the association between attachment avoidance and self-concept reorganization following marital separation. International Journal of Psychophysiology, 88(3), 253-260. doi: 10.1016/j.ijpsycho.2012.04.004.

Tarvainen, M. P., Niskanen, J. P., Lipponen, J. A., Ranta-Aho, P. O., \& Karjalainen, P. A. (2014). Kubios HRV - Heart rate variability analysis software. Computer Methods and Programs in Biomedicine, 113, 210-220. https://doi.org/10.1016/j.cmpb.2013. 07.024.

Task Force of the European Society of Cardiology, the North American Society of Pacing and Electrophysiology. (1996). Heart rate variability: standards of measurement, physiological interpretation, and clinical use. Circulation, 93, 1043-1065. doi: 10.1161/01.CIR.93.5.1043.

Thayer, J. F., Hansen, A. L., Saus-Rose, E., \& Johnsen, B. H. (2009). Heart rate variability, prefrontal neural function and cognitive performance: the neurovisceral integration perspective on selfregulation, adaptation, and health. Annals of Behavioral Medicine, 37, 141-153.

Thompson, R. A. (1994). Emotion regulation: A theme in search of definition. Monographs of the Society for Research in Child Development, 59(2-3), 25-52, 250-283. https://doi.org/10.1111/j.1540-5834.1994.tb01276.x. 
Tininenko, J. R., Measelle, J. R., Ablow, J. C., and High, R. (2012). Respiratory control when measuring respiratory sinus arrhythmia during a talking task. Biological Psychology, 89, 562569. doi: 10.1016/j.biopsycho.2011. 12.022 . 
Figure 1.

Differences in heart rate variability (root mean square of successive beat-to-beat interval differences; rMSSD) responses to and recovery from AAI in Disorganized/Unresolved $(n=15)$ and Organized/Resolved $(n=44)$ participants.

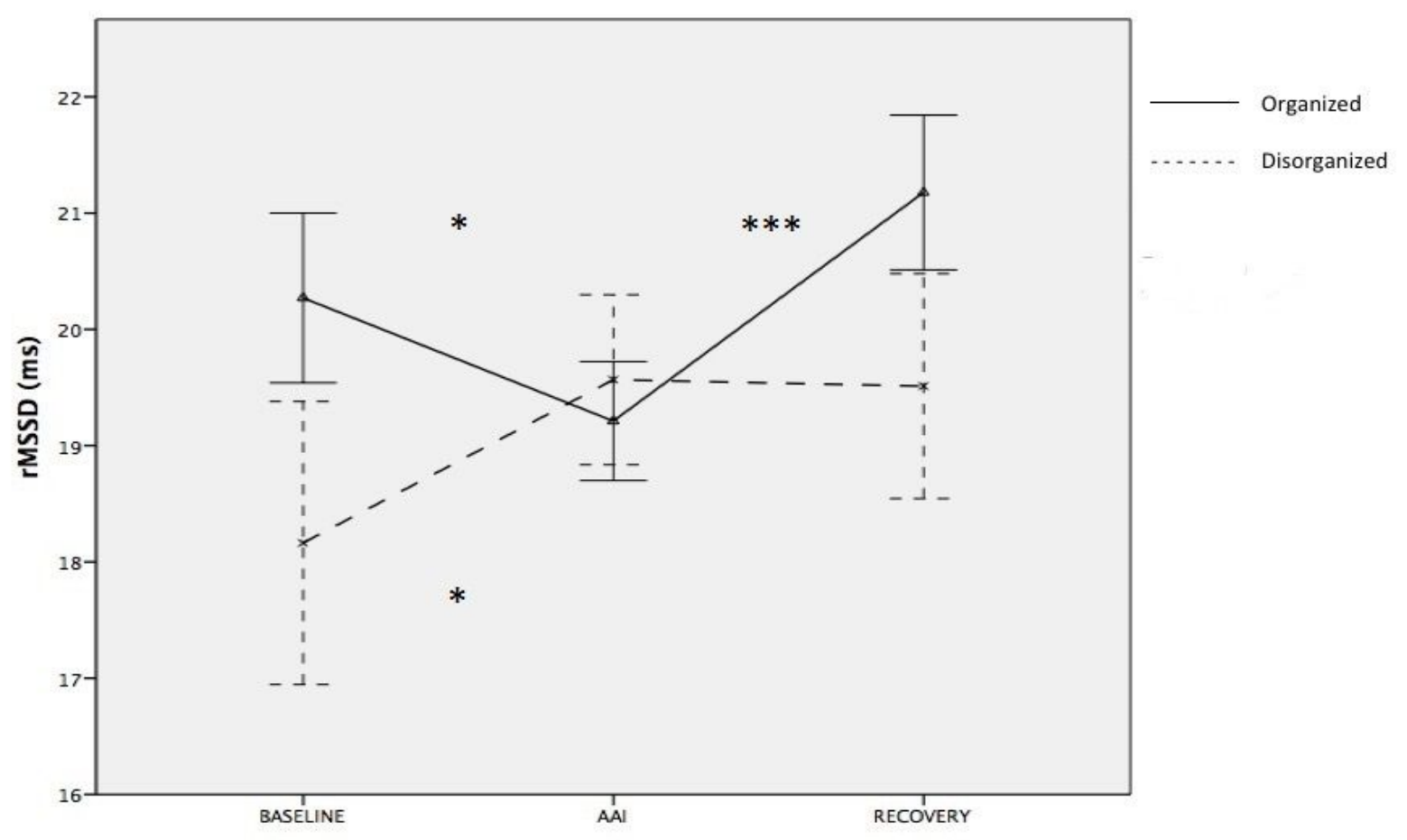

Notes: rMSSD $=$ root mean square of successive beat-to-beat interval differences; AAI $=$ Adult Attachment Interview; Error bars represent \pm 1 standard errors of the mean; ${ }^{*} p<.05 ;{ }^{* * *} p<.001$. 


\section{Table 1.}

Differences (means and standard deviation) between the group with Disorganized/Unresolved attachment and the group with Organized/Resolved attachment in the age, in the principal characteristics of the sample and physiological variables of the study.

\begin{tabular}{lccc}
\hline & Disorganized & Organized & $p$-value \\
& Participants & Participants & \\
& $(n=15)$ & $(n=44)$ & \\
\hline Age (years) & $32.4 \pm 11.6$ & $28.6 \pm 7.5$ & $t=1.47 ; p=.15$ \\
Sex (Males:Females) & $5: 10$ & $24: 20$ & $\chi^{2}=2.01 ; p=.17$ \\
Annual Income (Euros) & 24.822 & 25.334 & $t=1.15 ; p=.28$ \\
Years of education & 13.91 & 14.38 & $t=0.64 ; p=.43$ \\
Baseline HR (bpm) & $84.6 \pm 9.4$ & $83.5 \pm 13.3$ & $t=0.30 ; p=.77$ \\
Baseline vmHRV (ms) & $18.2 \pm 4.7$ & $20.3 \pm 4.8$ & $t=0.15 ; p=.15$ \\
HR during the AAI (bpm) & $79.3 \pm 9.1$ & $81.0 \pm 11.2$ & $t=0.53 ; p=.58$ \\
vmHRV during the AAI (ms) & $19.6 \pm 2.8$ & $19.2 \pm 3.4$ & $t=0.36 ; p=.72$ \\
HR during recovery (bpm) & $78.7 \pm 10.3$ & $77.8 \pm 11.1$ & $t=0.29 ; p=.77$ \\
vmHRV during recovery (ms) & $19.5 \pm 3.8$ & $21.2 \pm 4.4$ & $t=1.30 ; p=.19$ \\
\hline Notes: HR = Heart rate; HRV $=$ Root Mean Square Successive Difference; AAI & & \\
Interview. & & & \\
\hline
\end{tabular}

